Journal of Computer Science 8 (5): 694-700, 2012

ISSN 1549-3636

(C) 2012 Science Publications

\title{
Performance of Improved Directed Diffusion Protocol for Sensor Networks under Different Mobility Models
}

\author{
${ }^{1}$ Kannammal, K.E. and ${ }^{2}$ T. Purusothaman \\ ${ }^{1}$ Department of CSE, \\ Sri Shakthi Institute of Engineering and Technology, Coimbatore-62, India \\ ${ }^{2}$ Department of CSE, Government College of Technology, Coimbatore-13, India
}

\begin{abstract}
Problem statement: In sensor networks, some routing protocols performance differs under different mobility models. In ns2, there are two kinds of directed diffusion protocols, rate based gradient mechanism (diffusion/rate) and probability based gradient mechanism (diffusion/prob). In this research, we proposed a method to improve the performance of Directed diffusion/prob protocol by adding a new interest message propagation mechanism and analyzed the protocol under different mobility models. Approach: In this study, we describe a method for increasing the performance of diffusion/prob protocol and reduce the overhead with varying node speed and under different mobility models. We carried out the work using network simulator (ns2) and observed the performance improvement. Results: The performance of the proposed protocol is evaluated in terms of power consumption, overhead and routing load with Random way point, Random point group and Manhattan mobility models. Moreover, it is been compared with the normal diffusion/prob protocol. Conclusion: We have successfully implemented the proposed diffusion/prob protocol and compared it with other diffusion protocols with different metrics. We hereby conclude that it will be suitable for mobile scenario and the performance of the protocols are varied on different mobility models.
\end{abstract}

Key words: Wireless sensor networks, mobility model, node speed, density, gradient based mechanism, directed diffusion

\section{INTRODUCTION}

A Wireless Sensor Networks (WSN) is a group of specialized transducers with a communications infrastructure intended to watch and record conditions at diverse locations. Commonly monitored parameters are temperature, humidity, pressure, wind direction and speed, illumination intensity, vibration intensity, sound intensity, power-line voltage, chemical concentrations, pollutant levels and vital body functions (Akkaya and Younis, 2005; Akyildiz et al., 2002).

A sensor network consists of multiple detection stations called sensor nodes, each of which is small, lightweight and portable. Every sensor node is equipped with a transducer, microcomputer, transceiver and power source.

The transducer generates electrical signals based on sensed physical effects and phenomena. The microcomputer processes and stores the sensor output. The transceiver, which can be hard-wired or wireless, receives commands from a central computer and transmits data to that computer. The power for each sensor node is derived from the electric utility or from a battery.
The concept of MSN in the context of pervasive ubiquitous networks has emerged in recent years, although the genius of Marc Weiser envisaged this concept as early as in 1991(Weiser, 1991). MSNs the have the energy, processing limitations and similar architecture to WSN, but are supplemented with mechanisms that enable the devices to move in space over time. A MSN owes its name to the presence of mobile sink or sensor nodes within the network. The advantages of mobile wireless sensor network over static wireless sensor network are increased energy efficiency, improved coverage, enhanced target tracking and superior channel capacity (Munir et al., 2007). In MSN the channel capacity is more as compared to static WSN. The capacity has been calculated if mobile sink within WSN and has come out to be more times than static WSN, provided the number of mobile sink increases linearly with the growth of sensor nodes (Chen and Ma, 2006; Al-Karaki and Kamal, 2004). The other important features of mobile WSN are its better targeting and data fidelity. Because, mostly the sensors are deployed randomly,

Corresponding Author: Kannammal, K.E., Department of CSE, Sri Shakthi Institute of Engineering and Technology, Coimbatore-62, India 
therefore, there is often a requirement to move the sensor node for better sight or for close proximity. In addition, mobility helps in better quality of communication among mobile sensor nodes. In a sparse or disconnected network, this property is very much helpful to maintain connectivity between the nodes in the network.

Related works: WSN routing protocols are classified into three categories: flat routing protocols, hierarchical routing protocols and location based routing protocols. Generally, the flat routing protocols are simple, robust and well suited for small and mid-scale networks. It requires less power consumption because there is neither hierarchy nor additional power consumption for managing the clusters. While hierarchical routing protocols are, complex and well suited for large-scale networks. In location-based routing protocols, sensor nodes are communicated by their locations. The distance between neighbouring nodes can be calculated based on incoming signal strengths (Breslau et al., 2000).

\section{MATERIALS AND METHODS}

Directed diffusion: Directed Diffusion (DD) protocol, was proposed by Intanagonwiwat et al. (2003), is a data-centric and application aware paradigm in the sense that all data generated by sensor nodes are named by attribute-value pairs (Yen et al., 2008). It is data centric communication and query based protocol, where sink send queries to the sensors in an on-demand fashion by disseminating an interest. In ns2, there are two kinds of directed diffusion protocol implementation,rate based gradient mechanism (diffusion/rate) and probability based gradient mechanism (diffusion/prob) (Kannammal and T.purusothaman, 2011). Its operation is divided in to three stages: interest propagation, gradient setup and data delivery along reinforced path (Kulik et al., 2002).

Interest propagation: When sink node wants some information from source nodes it sends out its query to its neighbour sensor nodes. The corresponding query is carried by interest packet. The sensor node receives the interest packet can temporarily store the packet and search for all the matching target data.

Initial gradient setup: Using Gradient in DD, the data propagation direction is calculated with least cost principle. Propagation of interest packets setup the gradient in the network for delivering data to the sink. Gradient is a reply link to a neighbour node from which the interest was received.
Data delivery: In data propagation phase, source node sends data packets to sink node to the initial setup gradient direction. Sink sends a reinforced packet to the neighbour node which is the first one receiving the target data. The neighbour node that receives the reinforced packet can also reinforce and select the neighbour node that can receives the new data first. Consequently, a path with maximum gradient is formed, so that in future received data packets can transmitted along best-reinforced path. Finally, the source will send the required data, in selected path.

Mobility model: The mobility model is to describe the movement pattern of mobile nodes, location, velocity and acceleration change over time. Since mobility patterns play a significant role in determining the performance of the protocol, it is desirable for mobility models to emulate the movement pattern of targeted real life scenario in a reasonable way.

In the past history, the wireless network research community relied on simple mobility models such as random waypoint mobility model. However, this model is, widely accepted, too simple, very useful in analysis and simulation. Recently the researcher has started using the alternative mobility models with different mobility characteristics such as Manhattan Mobility Model, Gauss Markov Mobility Model, Random point group mobility.

Random way point mobility model: Imelinsky and Korth (1996) first proposed the Random Way Point model (RWP) (Jayakumar and Gopinath, 2008a). It is a simple, widely accepted 'benchmark' mobility model to evaluate the mobile adhoc routing protocols (Altman and Jimenez, 2003). This mobility model is included in the widely used network simulator ns-2 is shown in Fig. 1.

In this mobility model, at every instant, mobile node randomly selects one location in the simulation field as the destination. It then travels towards the destination with constant velocity chose uniformly and randomly from $[0, \mathrm{~V}]$, where the parameter $\mathrm{V}$ is the maximum allowable velocity for every mobile node (Breslau et al., 2000). The velocity and direction of a nodes are chosen independently of other nodes. After reaching the destination, the node stops for a duration defined by the 'pause time' parameter. If $\mathrm{T}=0$, this leads to continuous mobility. After this duration, it again chooses another random destination in the simulation field and moves towards it (Bettstetter and Wagner, 2002).

Reference point group mobility model: Random Point Group Mobility (RPGM) can used in military battlefield communication. This model exhibits spatial dependency. 


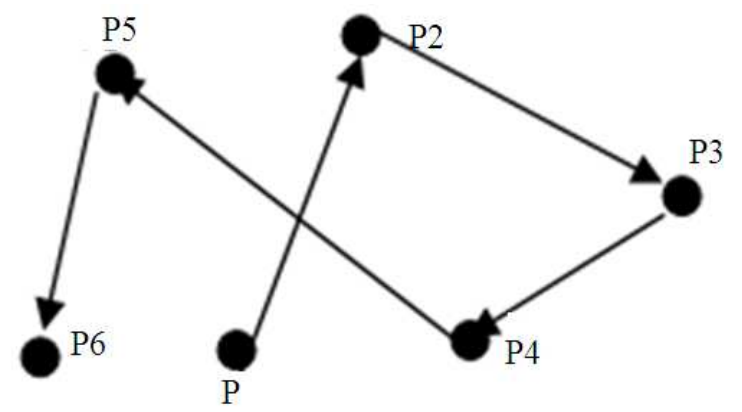

Fig. 1: Random way point model

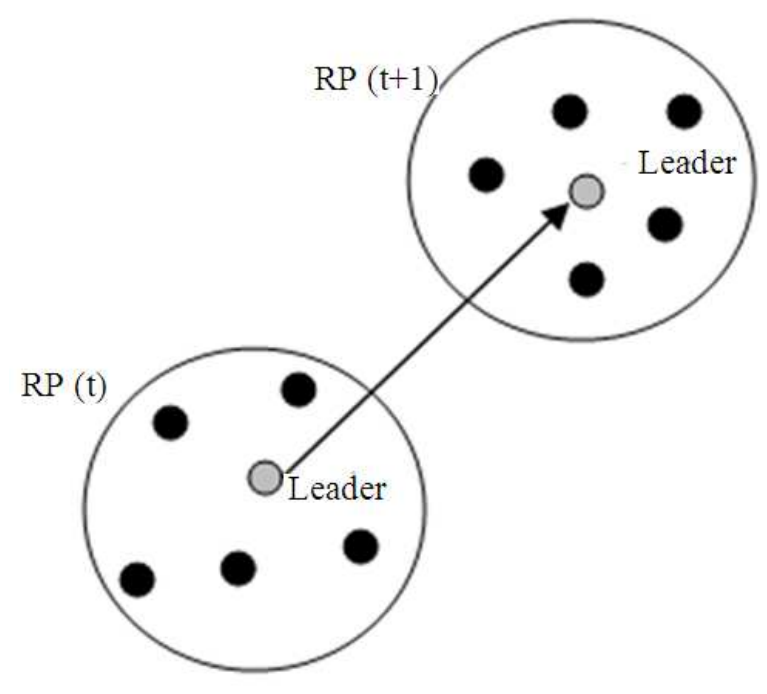

Fig. 2: Random point group mobility

In Fig. 2 shows this model, it consists of groups of nodes that work cooperatively. Each group has a group leader and number of members. The movement of the group leader determines the mobility behavior of the entire group. Motion of the group leader at time $t$ represented by the vector $\mathrm{V}$. Each member of this group deviates from this general motion vector $\mathrm{V}$ by some degree. For each node, mobility is assigned with a reference point that follows the group movement. The random motion is independent identically distributed random process whose length is uniformly distributed in the interval $\left[0, r_{\max }\right]$ where $r_{\max }$ is maximum allowed distance deviation and the direction is uniformly distributed in the interval $[0,2 \pi]$. Since the group leader mainly decides the mobility of group members, group mobility pattern is expected to have an high spatial dependence for small values of speed and angle deviation ratio (Jayakumar and Ganapathi, 2008b).
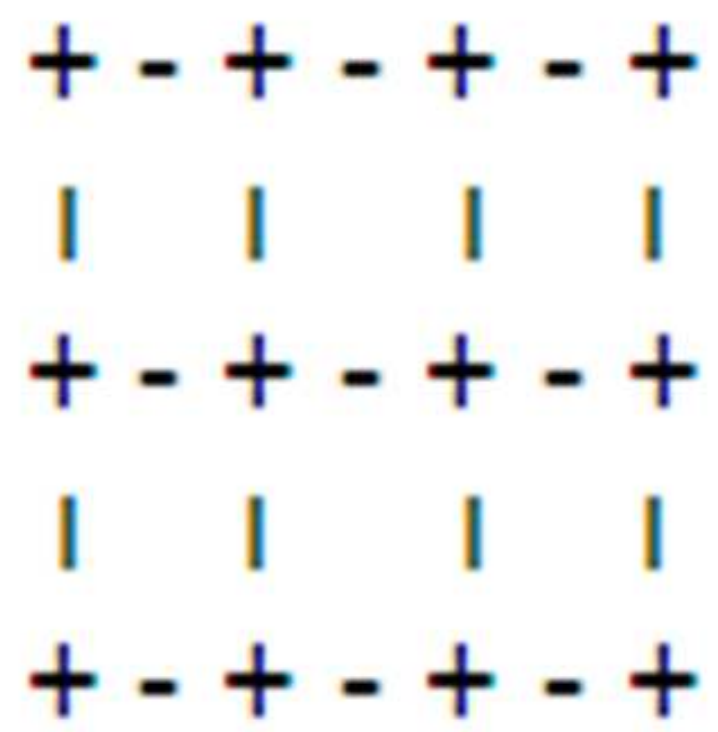

Fig. 3: Manhattan model

Manhattan mobility model: The Manhattan model (MAN) is to emulate the movement pattern of mobile nodes on streets. It can be useful in modelling movement in an urban area. In this mobility model, the mobile nodes move in horizontal or vertical direction in the terrain. This employs a probabilistic approach in the selection of nodes movements as at each intersection, node can move in left, right or straight in same direction. The probability of taking a left turn is $1 / 2$ and that of right turn is $1 / 4$ in each case. The mobile node is allowed to move along the grid of horizontal and vertical direction in the terrain, which is shown in Fig. 3.

The improved Directed diffusion: In the proposed method, a sensor node will propagates the interest message only if the condition is based on speed of the node and the density of nearby nodes are satisfied. If the interest message is allowed to propagate through a node $\mathrm{N}$ from $\mathrm{X}$, then the node is a member of the routing path. After the completion of the route resolving process, the destination sensor node $\mathrm{Z}$ will have a path through the node $\mathrm{N}$. If the interest message is not allowed to propagate through a node $\mathrm{N}$ from $X$, then the node is not a member of the routing path. After the completion of the route resolving process, the destination sensor node $\mathrm{Z}$ will not have a path through the node $\mathrm{N}$ (Kannammal and T.purusothaman, 2012).

In the proposed method, if a node receives a packet then the node will check the Packet Pi that is a new packet or not. If the received packet is not a new one 
then the node will recheck the packet is already been forwarded. If that is the case then the packet will be dropped, on the contrary if the packet is not forwarded then the interest timer is updated.

If the packet is new and it is an interest packet then the number of adjacent nodes and mobility factors are calculated. $\mathrm{Ni}$ is number of nearby nodes for a particular node; the mobility factor is the ratio of node speed and the expected node speed. If $\mathrm{Ni}$ is less than minimum expected Neighbours then propagate the interest packets into the network. Else if the Mobility factor is less than mobility threshold then propagate the interest packets into the Network. If both the conditions are not satisfied then it will not propagate the interest. If the received packet is not an interest packet then it is handled normally.

A node $\mathrm{X}$ starts a route resolve process for finding a path to $\mathrm{Z}$. Any intermediate node $\mathrm{N}$ receiving interest message will do the following:

On receiving a Directed Diffusion Packet Pi:

1. If ( $\mathrm{Pi}$ is new)

2. If ( $\mathrm{Pi}$ is of type INTEREST)

// Resolve No. of neighbors Ni

// Resolve Node Speed Si

//Mobility Factor $\mathrm{Mi}=\mathrm{Si} / \mathrm{Se}$

3. If ( $\mathrm{Ni}<$ MinExpectedNeighbors)

4. Propagate the interest

5. Else if (Mi < Mobility Threshold)

6. Propagate the interest

7. Else do not propagate the Interest

8. End

9. Else Handle it normally

10. End

11. Else Handle then normally // Other Packet types

12. End

13. Else if Drop the packets

14. Else Update the Interest Timer

15. End

16 End

Where:

- Mobility Threshold is a value between 0 and 1 , which will control the behavior of the algorithm

- Expected Node speed (Se) may be chosen with respect to the average maximum speed of the nodes

- Minimum Expected Neighbors can be decided with respect to the average network density of the network under consideration

\section{RESULTS AND DISCUSSION}

In this study, we examine the impact of different mobility models on the performance of normal and proposed directed diffusion/prob. The routing protocol used for the simulation is available with ns-2. For each of these scenarios, movements were generated using software called Bonnmotion is java software, which creates and analyses mobility scenarios. It is developed within the communication systems group at the institute of computer science of the University of Bonn, Germany, where it serves as a tool for the investigation of mobile ad hoc network characteristics. The scenarios can also be exported for the network simulators ns-2, ns-3, GloMoSim/QualNet, COOJA, MiXiM and ONE.

The simulations are conducted $100 \mathrm{sec}$ for each protocol and the simulated mobility network area is 800 $\times 800 \mathrm{~m}$ rectangle. We have evaluated these sensor network protocols in different node speed: 5, $10,15,20,25 \mathrm{~m} \mathrm{sec}^{-1}$ with 30 nodes.

Performance metrics: For the simulation results, three performance metrics have being used in our simulations as shown below:-

Power consumption: Average power consumption of the nodes in the sensor network.

Routing load: The ratio of the number of routing messages propagated by every node in the network and the number of data packets successfully delivered to all destination nodes.

Overhead: It is measured in terms of total no. of generated and forward routing messages at routing layer.

Experimental results: Simulation results for the two routing protocols is detailed below .

In Fig. 4, graph shows that the total dropped packets by normal and proposed diffusion/prob and with varying node speed using random way point mobility model. As a result, this proves the proposed diffusion /prob performed well and number of dropped packets is less compared with normal diffusion/prob.

In Fig. 5, the graph shows that the routing load of the entire network with respect to node speed for proposed and normal diffusion/prob with RWP. The entire routing load in proposed diffusion/prob is higher than normal diffusion/prob.

In Fig. 6, the graph shows that the overhead of the entire network with respect to node speed for proposed and normal diffusion/prob. The proposed diffusion/prob produces less overhead and increase the overall performance. 


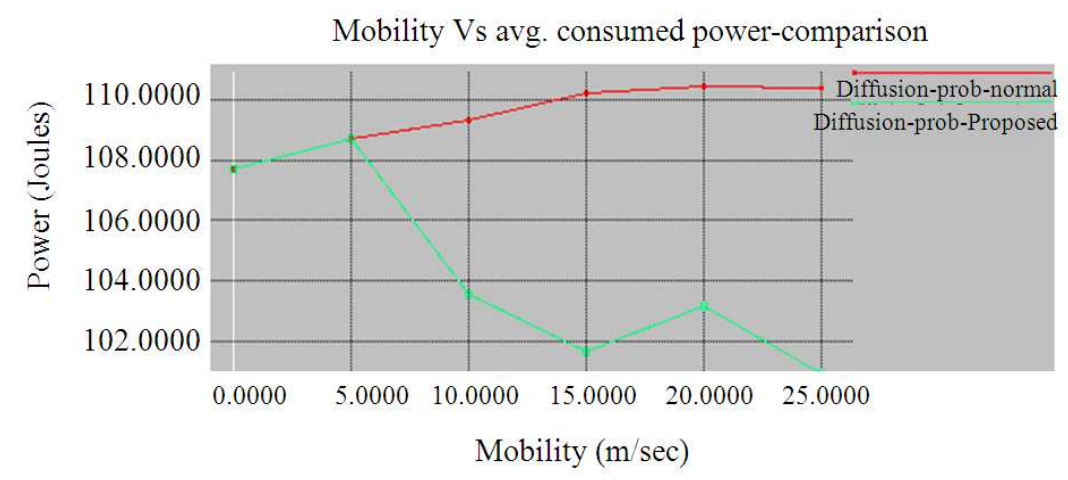

Fig. 4: Consumed power Vs mobility with RWP

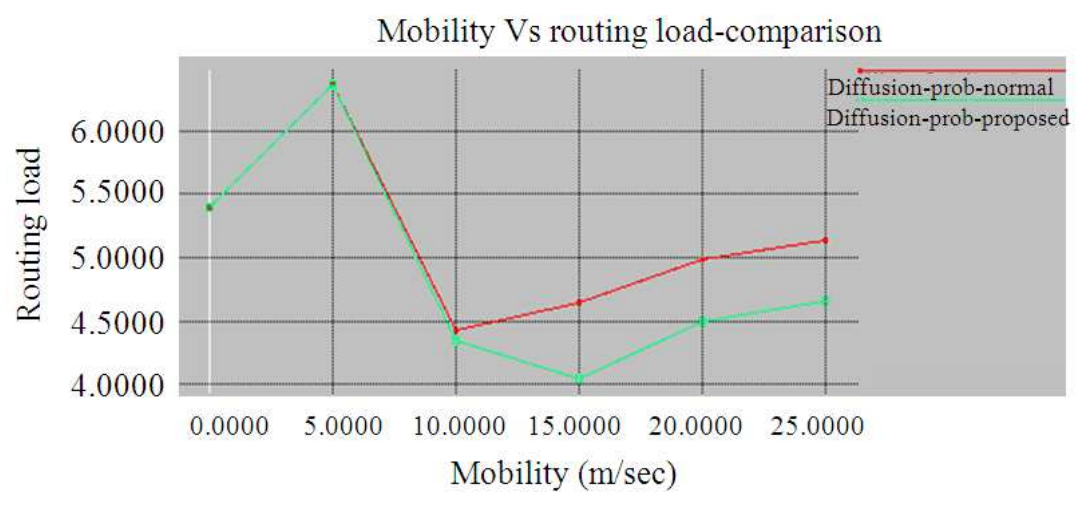

Fig. 5: Routing load Vs mobility with RWP

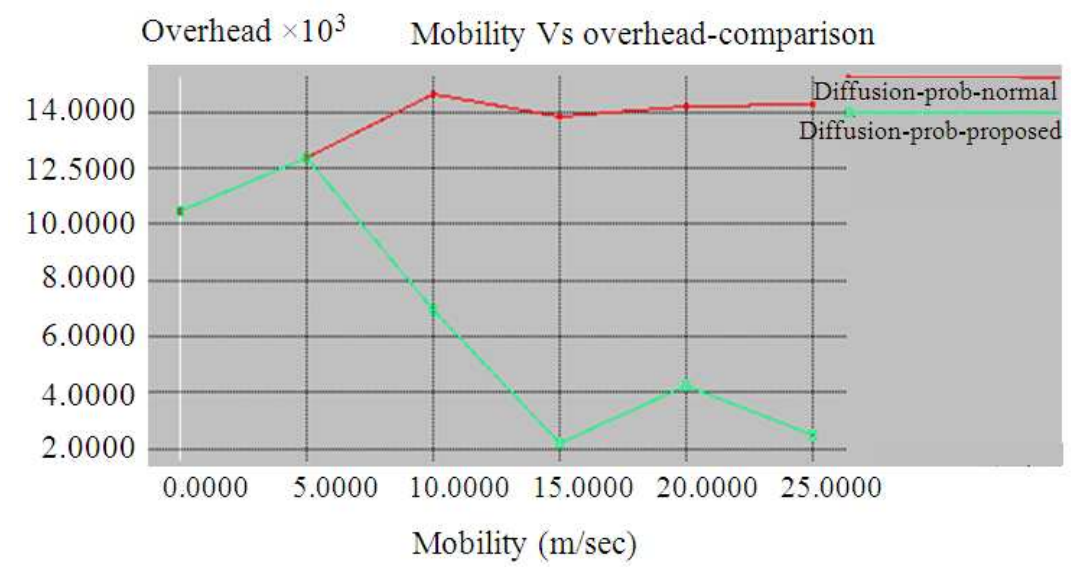

Fig. 6: Overhead Vs mobility with RWP

This is because here not all the nodes are involved in broadcasting the interest message, the node will only forward a message based on nearby node density as well as the mobility threshold value.

In Fig. 7-9, the graph shows the power consumption, routing load and overhead with different mobility models. Both the normal and proposed diffusion/prob performs well under RWP mobility model compared with RPGM and MAN mobility models in terms of power consumption, routing load and overhead. Proposed diffusion/rate performs well under all the three mobility models. 


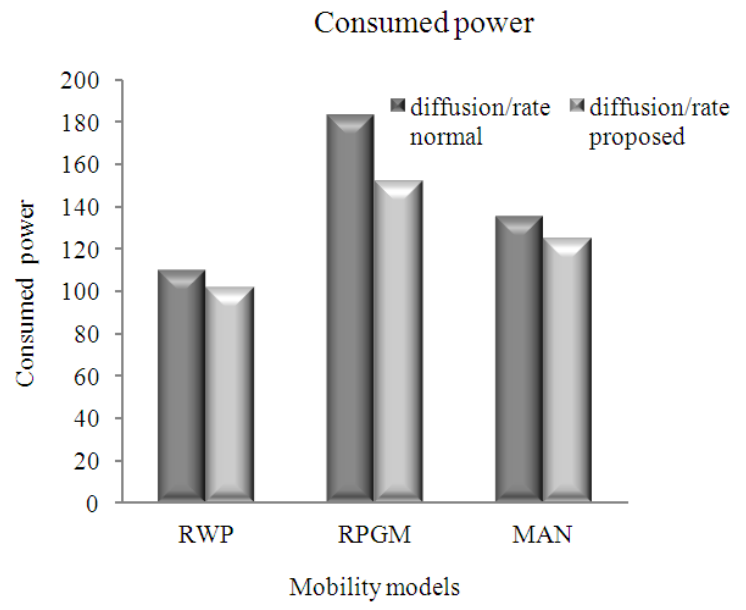

Fig. 7: Consumed power Vs mobility models

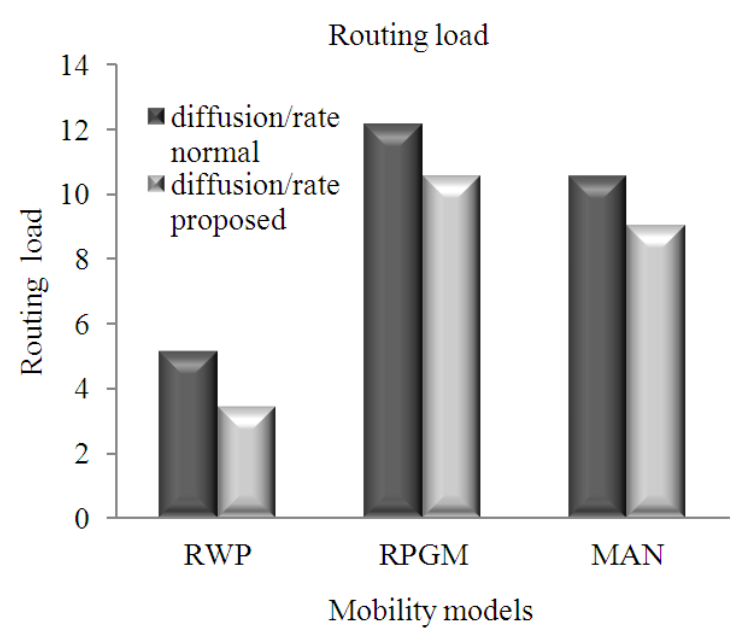

Fig. 8: Routing load Vs mobility models

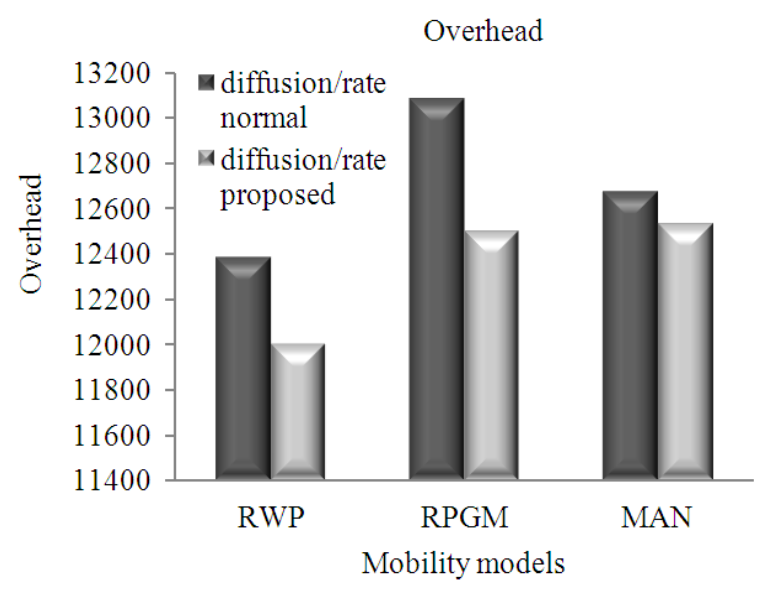

Fig. 9: Overhead Vs mobility models

\section{CONCLUSION}

In this study, we have highly improved the performance of diffusion/prob by implementing mobility and density aware interest propagation mechanism. Also evaluated the normal and proposed diffusion/prob protocol under different mobility models.

From the results obtained, the proposed algorithm increases the performance even in the case of mobile scenarios. Therefore, we hereby conclude that proposed algorithm is very well recommended for mobile and very dense networks. There are similar variety of different models of directed diffusion protocols available based on data propagation and gradient filter mechanisms. In this work, we have emphasized only the diffusion/prob protocol by using new mobility and density aware interest propagation mechanism and analyzed it with different mobility models. Future works can be carried out on the issues in applying the proposed mechanism in other directed diffusion protocols and those can be analyzed under different mobility models.

\section{REFERENCES}

Akkaya, K. and M. Younis, 2005. A survey on routing protocols for wireless sensor networks. Ad Hoc Netw., 3 3: 325-349. 10.1016/j.adhoc.2003.09.010

Akyildiz, I.F., W. Su, Y. Sankarasubramaniam and E. Cayirci, 2002. Wireless sensor networks: A survey. J. Comput. Netw., 38: 393-422. DOI: 10.1016/S1389-1286(01)00302-4

Al-Karaki, J.N. and A.E. Kamal, 2004. Routing techniques in wireless sensor networks: A survey. IEEE Wireless Commun., 11: 6-28. DOI: 10.1109/MWC.2004.1368893

Altman, E. and T. Jimenez, 2003. NS Simulator for Beginners. University of de Los Andes, Merida, Venezuela and ESSI, Sophia Antipolis, France.

Bettstetter, C. and C. Wagner, 2002. The spatial node distribution of the random waypoint mobility model. Institute of Communication Networks, Germany.

Breslau, L., D. Estrin, K. Fall, S. Floyd and J. Heidemann et al., 2000. Advances in network simulation. IEEE Comput., 33: 59-67. DOI: $10.1109 / 2.841785$

Chen, C. and J. Ma, 2006. MEMOSEN: Multi-radio enabled mobile wireless sensor network. Proceedings of the 20th International Conference on Advanced Information Networking and Applications, Apr. 18-20, IEEE Xplore Press, pp: 5-5. DOI: 10.1109/AINA.2006.213 
Imelinsky, T. and H. Korth, 1996. Mobile Computing. 1st Edn., Springer, Boston, ISBN: 0792396979, pp: 728.

Intanagonwiwat, C., R. Govindan, D. Estrin, J. Heidemann and F. Silva, 2003. Directed diffusion for wireless sensor networking. IEEE/ACM Trans. Netw., $\quad 11: \quad 2-16 . \quad$ DOI: 10.1109/TNET.2002.808417

Jayakumar, G. and G. Ganapathi, 2008b. Reference point group mobility and random waypoint models in performance evaluation of MANET routing protocols. J. Comput. Syst. Netw. Commun., 2008: 10-10. DOI: $10.1155 / 2008 / 860364$

Jayakumar, G. and G. Gopinath, 2008a. Performance comparison of two on-demand routing protocols for ad-hoc networks based on random way point mobility model. Am. J. Applied Sci., 6: 659-664. DOI: 10.3844/ajassp.2008.659.664

Kannammal, K.E. and T. Purusothaman, 2011. Comparison of data centric routing protocols with random way point mobility model in mobile sensor networks. Eur. J. Sci. Res., 65: 546-552.

Kannammal, K.E. and T. Purusothaman, 2012. New interest propagation mechanism in directed diffusion protocol for mobile sensor networks. Eur. J. Sci. Res., 68: 36-42.
Kulik, J., W. Heinzelman and H. Balakrishnan, 2002. Negotiation-based protocols for disseminating information in wireless sensor networks. J. Wireless Netw., 8: 169-185. DOI: 10.1023/A:1013715909417

Munir, S.A., B. Ren, W. Jiao, B. Wang and D. Xie et al., 2007. Mobile wireless sensor network: architecture and enabling technologies for ubiquitous computing. Proceedings of the 21st International Conference on Advanced Information Networking and Applications Workshops, May 2123, IEEE Xplore Press, Niagara Falls, Ont., pp: 113-120. DOI: 10.1109/AINAW.2007.257

Weiser, M., 1991. The computer for the twenty-first century. Scientific American.

Yen, W., C.W. Chen and C.H. Yang, 2008. Single gossiping with directional flooding routing protocol in wireless sensor networks. Proceedings of the 3rd IEEE Conference on Industrial Electronics and Applications, Jun. 3-5, IEEE Xplore Press, Singapore, pp: 1604-1609. DOI: 10.1109/ICIEA.2008.4582790 